\title{
THE SIGNED TOTAL ROMAN $k$-DOMATIC NUMBER OF A GRAPH
}

\author{
Lutz Volkmann \\ Lehrstuhl II für Mathematik \\ RWTH Aachen Univertsity \\ 52056 Aachen, Germany \\ e-mail: volkm@math2.rwth-aachen.de
}

\begin{abstract}
Let $k \geq 1$ be an integer. A signed total Roman $k$-dominating function on a graph $G$ is a function $f: V(G) \longrightarrow\{-1,1,2\}$ such that $\sum_{u \in N(v)} f(u) \geq k$ for every $v \in V(G)$, where $N(v)$ is the neighborhood of $v$, and every vertex $u \in V(G)$ for which $f(u)=-1$ is adjacent to at least one vertex $w$ for which $f(w)=2$. A set $\left\{f_{1}, f_{2}, \ldots, f_{d}\right\}$ of distinct signed total Roman $k$-dominating functions on $G$ with the property that $\sum_{i=1}^{d} f_{i}(v) \leq k$ for each $v \in V(G)$, is called a signed total Roman k-dominating family (of functions) on $G$. The maximum number of functions in a signed total Roman $k$-dominating family on $G$ is the signed total Roman k-domatic number of $G$, denoted by $d_{s t R}^{k}(G)$. In this paper we initiate the study of signed total Roman $k$-domatic numbers in graphs, and we present sharp bounds for $d_{s t R}^{k}(G)$. In particular, we derive some Nordhaus-Gaddum type inequalities. In addition, we determine the signed total Roman $k$-domatic number of some graphs.
\end{abstract}

Keywords: signed total Roman $k$-dominating function, signed total Roman $k$-domination number, signed total Roman $k$-domatic number.

2010 Mathematics Subject Classification: 05C69.

\section{REFERENCES}

[1] E.J. Cockayne and S.T. Hedetniemi, Towards a theory of domination in graphs, Networks 7 (1977) 247-261.

doi:10.1002/net.3230070305

[2] T.W. Haynes, S.T. Hedetniemi and P.J. Slater, Fundamentals of Domination in Graphs (Marcel Dekker, Inc., New York, 1998).

[3] E.A. Nordhaus and J.W. Gaddum, On complementary graphs, Amer. Math. Monthly 63 (1956) $175-177$.

doi: $10.2307 / 2306658$ 
[4] P.J. Slater and E.L. Trees, Multi-fractional domination, J. Combin. Math. Combin. Comput. 40 (2002) 171-181.

[5] L. Volkmann, Signed total Roman domination in graphs, J. Comb. Optim. 32 (2016) 855-871. doi:10.1007/s10878-015-9906-6

[6] L. Volkmann, Signed total Roman k-domination in graphs, J. Combin. Math. Combin. Comput., to appear.

[7] L. Volkmann, On the signed total Roman domination and domatic numbers of graphs, Discrete Appl. Math. 214 (2016) 179-186. doi:10.1016/j.dam.2016.06.006

[8] C. Wang, The signed k-domination number in graphs, Ars Combin. 106 (2012) 205-211.

Received 14 March 2016

Revised 23 August 2016

Accepted 23 August 2016 